\title{
Dark Matter Signals from Timing Spectra at Neutrino Experiments
}

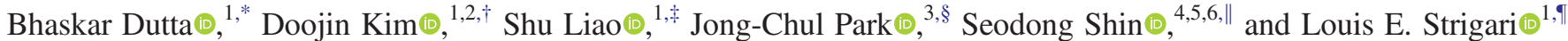 \\ ${ }^{1}$ Mitchell Institute for Fundamental Physics and Astronomy, Department of Physics and Astronomy, \\ Texas A\&M University, College Station, Texas 77843, USA \\ ${ }^{2}$ Department of Physics, University of Arizona, Tucson, Arizona 85721, USA \\ ${ }^{3}$ Department of Physics, Chungnam National University, Daejeon 34134, Republic of Korea \\ ${ }^{4}$ Department of Physics and IPAP, Yonsei University, Seoul 03722, Republic of Korea \\ ${ }^{5}$ Center for Theoretical Physics of the Universe, Institute for Basic Science, Daejeon 34126, Republic of Korea \\ ${ }^{6}$ Department of Physics, Jeonbuk National University, Jeonju, Jeonbuk 54896, Republic of Korea
}

(Received 30 July 2019; revised manuscript received 13 February 2020; accepted 28 February 2020; published 26 March 2020)

\begin{abstract}
We propose a novel strategy to search for new physics in timing spectra at low-energy neutrino experiments using a pulsed beam, envisioning the situation in which a new particle comes from the decay of its heavier partner with a finite particle width. The timing distribution of events induced by the dark matter (DM) candidate particle scattering at the detector may populate in a relatively narrow range, forming a "resonancelike" shape. Because of this structural feature, the signal may be isolated from the backgrounds, in particular when the backgrounds are uniformly distributed in energy and time. For proof of the principle, we investigate the discovery potential for DM from the decay of a dark photon in the ongoing COHERENT experiment and show the exciting prospects for exploring the associated parameter space with this experiment. We analyze the existing CsI detector data with a timing cut and an energy cut, and we find, for the first time, an excess in the timing distribution that can be explained by such DM. We compare the sensitivity to the kinetic mixing parameter $(\epsilon)$ for current and future COHERENT experiments with the projected limits from LDMX and DUNE.
\end{abstract}

DOI: 10.1103/PhysRevLett.124.121802

Numerous theoretical and experimental ideas have been put forth to identify the mass and associated interactions of dark matter (DM) candidate particles. Since traditional weakly interacting massive particle (WIMP)-based searches have not yet detected DM [1], expanding the search of parameter space is well justified [2]. Many models of light $\mathrm{DM}(\lesssim \mathrm{GeV})$ emerge from a hidden or visible sector where light mediators (e.g., a dark photon) interact with DM [3-9]. Because the DM mass is light in these models, it is difficult to detect such DM in traditional WIMP-based direct detection experiments.

In this Letter, we develop a novel strategy to search for light DM that couples with light mediators and apply it to the data from the ongoing COHERENT experiment [10]. COHERENT makes use of a proton beam that impinges on a $\mathrm{Hg}$ target at the Spallation Neutron Source (SNS). Among the produced pions, $\pi^{+}$decays create prompt muon neutrinos and delayed antimuon and electron neutrinos. The measured energy spectra have been used to investigate new physics associated with neutrino nonstandard interactions

Published by the American Physical Society under the terms of the Creative Commons Attribution 4.0 International license. Further distribution of this work must maintain attribution to the author(s) and the published article's title, journal citation, and DOI. Funded by SCOAP.
(NSIs) [11,12] due to heavy or light mediators [13-20], generalized scalar and vector neutrino interactions [21], hidden sector models [22], and sterile neutrinos [23,24]. It also sets independent constraints on the effective neutron size distribution of CsI [25-27]. Since the proton beam is pulsed, the measured timing spectra may be used to distinguish between prompt and delayed events. The combined timing and energy spectra have been utilized to understand new physics models with neutrino flavor-dependent NSIs [28].

We show how both the timing and energy data from the COHERENT experiment can be used to search for light, $\lesssim 1 \mathrm{GeV}$, DM. The DM event under consideration is initiated by the production of a dark photon decaying into a pair of DM particles (e.g., Refs. [29,30]). A DM particle would then induce a nuclear recoil event at the detector. The dark photon production can occur from both $\pi^{-}$and $\pi^{0}$. Most of the $\pi^{-}$are stopped inside the $\mathrm{Hg}$ target and can create a dark photon via the absorption process, $\pi^{-}+p \rightarrow n+A^{\prime}$, followed by the decay of the dark photon $A^{\prime}$ to a DM pair [29]. The dark photon is emitted isotropically in this $\pi^{-}$absorption process. The $\pi^{0}$ may produce an ordinary photon and $A^{\prime}$ [30]. Since the $\pi^{0}$ move somewhat relativistically, the resulting DM lies relatively in the forward direction. Nevertheless, we find that the DM flux reaching the COHERENT detectors, which are located 
$\sim 90^{\circ}$ from the beam direction [10] (see Supplemental Material [31]), is comparable to that from the $\pi^{-}$absorption. Further, there are additional contributions from $\pi^{ \pm}+p / n \rightarrow n / p+\pi^{0}$.

The method that we develop to search for DM utilizes both the energy and timing spectra of the DM-initiated nuclear recoil events. We focus on the timing and energy spectra for the DM produced from the $\pi^{-}$absorption and the $\pi^{0}$ decay. In the COHERENT experiment, the $\pi^{-}\left(\pi^{+}\right)$ and $\pi^{0}$ abundances per proton on target are $0.05(0.11)$ and 0.1 , respectively $[29,32,33]$. The produced $A^{\prime}$ is mostly relativistic unless its mass is $\sim 138 \mathrm{MeV}$.

We first derive the timing spectrum of DM-induced nuclear recoil events along with their energy distribution and then compare the DM case to that of standard model (SM) neutrinos. The signal under consideration is initiated by production of a dark photon $A^{\prime}$ from the decay of the $\pi^{-}-p$ mesic state and $\pi^{0}$ decay through kinetic mixing. $A^{\prime}$ production and its subsequent decay to DM $\chi$ are governed by the following interaction Lagrangian:

$$
\mathcal{L}_{\text {int }} \supset g_{\chi} A_{\mu}^{\prime} \bar{\chi} \gamma^{\mu} \chi+e_{q} \epsilon_{1}^{q} A_{\mu}^{\prime} \bar{q} \gamma^{\mu} q
$$

where $e_{q}=e Q_{q}, g_{\chi}$ and $\epsilon_{1}^{q}$ are dark-sector gauge coupling and a kinetic mixing parameter (associated with the mixing between the $\gamma$ and new gauge boson $(\epsilon / 2) F^{\mu \nu \prime} F_{\mu \nu}$ [34-36]), respectively. This generic-looking Lagrangian can be accommodated in the context of a model, e.g., $[9,29]$.

Let us suppose that $A^{\prime}$ is produced at $t_{F}$ where $t_{F}$ is the timing of $\pi^{-, 0}$ production induced by the $1 \mathrm{GeV}$ SNS beam, which is $0.6 \mu$ s wide and pulsed at $60 \mathrm{~Hz}$. We then assume that $A^{\prime}$ flies for $v_{A^{\prime}}\left(t-t_{F}\right)$ along the $\theta$ direction with respect to the line joining the $\mathrm{Hg}$ target and the detector (see Supplemental Material [31]) and decays to a $\chi$ pair. One of the $\chi$ 's then may travel toward the detector for $v_{\chi} t^{\prime}$. Denoting the timing measured at the detector by $T$, we see that $T$ is the sum of $t$ and $t^{\prime}$, i.e., $T=$ $t+t^{\prime}\left[v_{A^{\prime}}\left(t-t_{F}\right), t-t_{F}, \cos \theta\right]$, where we explicitly express $t^{\prime}$ as a function of $t-t_{F}$ and $\cos \theta$. We are interested in the differential number of events in $T$ or, equivalently, the DM flux at the detector of interest, $f(T)=d N_{\chi} / d T$. Parametrizing the angular distribution of dark photons by $g(\cos \theta)$, we find $\left[\left(d^{2} N_{A^{\prime}}\right) /(d t d \cos \theta)\right]=g(\cos \theta)\left(1 / \tau_{A^{\prime}}\right) e^{-\left[\left(t-t_{F}\right) / \tau_{A^{\prime}}\right]} \Theta\left(t-t_{F}\right)$, where $\Theta(x)$ is the step function. $g(\cos \theta)$ is $1 / 2$ for dark photons from the $\pi^{-}$absorption. We then obtain $f(T) \propto \int d \cos \theta|(d T / d t)|^{-1}\left[\left(d^{2} N_{A^{\prime}}\right) /(d t d \cos \theta)\right]$. A simple geometry consideration gives $T=t+$ $v_{\chi}^{-1} \sqrt{x_{0}^{2}+v_{A^{\prime}}^{2}\left(t-t_{F}\right)^{2}-2 x_{0} v_{A^{\prime}}\left(t-t_{F}\right) \cos \theta}$, with $x_{0}$ being the distance between the $\mathrm{Hg}$ target and the detector. We consider both $\pi^{-}$and $\pi^{0}$ contributions. In our calculation, we use the GEANT4 [37] simulations for the COHERENT geometry to determine the angular and energy spectra of
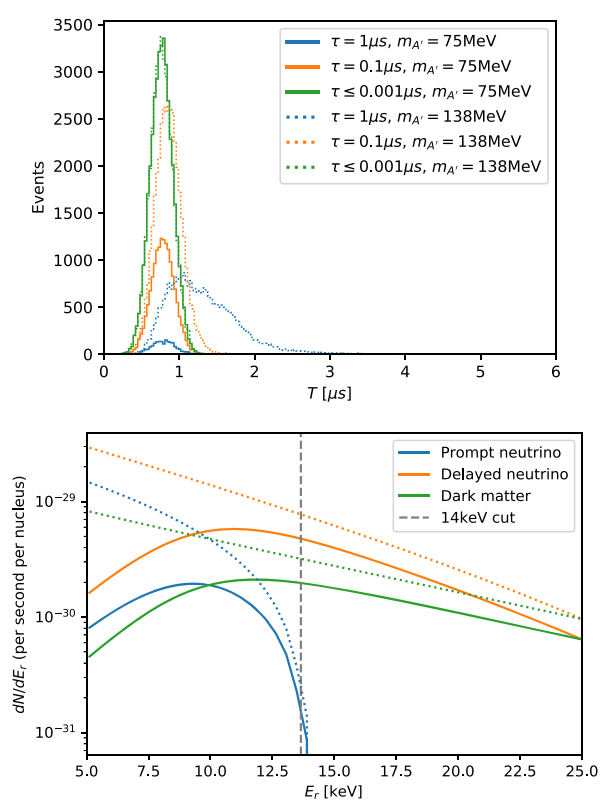

FIG. 1. (Top) Timing spectra of DM signal with three different values for $\tau_{A^{\prime}}$, in a relativistic $A^{\prime}$ scenario (solid) and a nonrelativistic $A^{\prime}$ scenario (dashed). (Bottom) Nuclear recoil spectrum produced from neutrino and DM interactions with (solid) and without (dashed) experimental efficiencies. The vertical dashed line indicates the energy cut that is used to eliminate prompt $\nu$-induced events.

photons from pion absorption and decays [33]. We find that the $\pi^{0}$ contribution is bigger than the $\pi^{-}$absorption.

The top panel of Fig. 1 demonstrates example timing spectra for a CsI detector, with three different choices for the rest-frame mean lifetime of $A^{\prime}$. The solid and dashed histograms are for a relativistic dark photon $\left(m_{A^{\prime}}=75 \mathrm{MeV}\right)$ and a nonrelativistic dark photon $\left(m_{A^{\prime}}=138 \mathrm{MeV}\right)$, respectively, with $m_{\chi}$ fixed to $5 \mathrm{MeV}$. Here the $\pi^{-}$flux - which is approximated by a Gaussian distribution with a mean value of $0.7 \mu$ s and a width of $0.15 \mu \mathrm{s}$ to model the arriving time of the proton on target, which reproduces the timing spectrum in [32]-is convoluted. For the nonrelativistic case, most of the $\chi$ 's can reach the detector [modulo a factor of $\left(4 \pi x_{0}^{2}\right)^{-1}$ ]. Not surprisingly, as $A^{\prime}$ is shorter lived, the spectrum width gets narrower, manifesting in a resonancelike bump feature more visibly. By contrast, for the relativistic case, if $A^{\prime}$ is long-lived, it decays far away from the detector so that only a small fraction of the $\chi$ 's can reach the detector, contributing to the upper tail of the spectrum. Therefore, relatively short-lived $A^{\prime}$ would give more statistics. Indeed, we see that most of DM events populate within $\sim 1.5 \mu \mathrm{s}$, which roughly corresponds to the mean value plus the width of the beam pulse. Note that prompt neutrinos leave events within $\sim 1.5 \mu \mathrm{s}$, whereas delayed neutrinos spread out over a broad range [10,32]. So, requiring $T \lesssim 1.5 \mu \mathrm{s}$ essentially rejects most of delayed neutrino events, while a large portion of prompt neutrino events and relativistic 
(nonrelativistic) $A^{\prime}$-induced DM events irrespective of $\tau_{A^{\prime}}$ (with $\tau_{A^{\prime}} \lesssim 0.1 \mu \mathrm{s}$ ) are kept.

Regarding DM-nucleus scattering, we remark that, in principle, DM scattering can be governed by physics different from that for dark photon production encoded in Eq. (1). Introducing a generic mediator of mass $M^{\prime}$, DMmediator coupling $g_{D}$, and quark-mediator coupling $e_{q} \epsilon_{2}^{q}$, we find that the differential spectrum in recoil energy $E_{r}$ of the target nucleus can be expressed as

$$
\begin{aligned}
\frac{d \sigma}{d E_{r}}= & \frac{e^{2}\left(\epsilon_{2}^{q}\right)^{2} g_{D}^{2} Z^{2}\left|F\left(2 m_{N} E_{r}\right)\right|^{2}}{4 \pi p_{\chi}^{2}\left(2 m_{N} E_{r}+M^{\prime 2}\right)^{2}} \\
& \times\left\{2 E_{\chi}^{2} m_{N}\left(1-\frac{E_{r}}{E_{\chi}}-\frac{m_{N} E_{r}}{2 E_{\chi}^{2}}\right)+E_{r}^{2} m_{N}\right\},
\end{aligned}
$$

where $F$ denotes the form factor, and where $Z$ and $m_{N}$ are the atomic number and the mass of the target nucleus. The underlying interaction is of dark photon type for illustration. We neglected $m_{\chi}$ in the curly brackets as $m_{N} \gg m_{\chi}$. Clearly, the spectral behavior is (nearly) independent of $m_{\chi}$. The bottom panel of Fig. 1 displays the expected nuclear recoil spectrum for $\left(M^{\prime}, m_{\chi}\right)=(75,5) \mathrm{MeV}$ (green). For comparison, we show the $E_{r}$ distributions of the prompt neutrinos (blue) and the delayed neutrinos (orange) with (solid) and without (dashed) experimental efficiencies. We see that prompt neutrino events occur almost entirely in the region $E_{r} \lesssim 14 \mathrm{keV}$, so employing a lower cut at this energy removes the remaining prompt neutrinos, while retaining a large portion of the DM candidate events.

In order to analyze the COHERENT data using both the energy and timing spectra [32] from neutrinos and DM, we adopt the statistical method described in Ref. [28]. We allow for Poisson fluctuations of the background in each energy and time bin [model (c) of Ref. [28] ], and fix the size of the neutron distribution to $R_{n}=4.7 \mathrm{fm}$. We also quote our results for $R_{n}=5.5 \mathrm{fm}$, which is the modelindependent central value obtained from the fit to the COHERENT data [38]. We examine two limiting cases: (i) the specific part of the energy and timing data in which the DM signal is predicted to appear, after removing as many neutrino-induced events as possible, and (ii) the full energy and timing data.

As discussed in the previous section, we apply cuts $E_{r}>14 \mathrm{keV}$ (16 photoelectrons) and $T<1.5 \mu$ s to substantially suppress both prompt and delayed neutrino events, but keep the DM candidate events [39] for the published COHERENT data [32]. We also apply an uppercut $E_{r}<26 \mathrm{keV}$ since the background is well understood for COHERENT in the range 5-26 keV [32]. The experimental efficiency is also given in [32]. After these cuts, we find 97 total events. Out of them, 49 events have been classified as the steady-state (SS) background, while 19 may be identified as delayed neutrino events forming the SM (i.e., neutrino) background. There are also three events in the cut window arising from beam related neutron (BRN) backgrounds. There is then an "excess" of 26 events, which corresponds to a $2.4 \sigma$ statistical uncertainty. For $R_{n}=5.5 \mathrm{fm}$, the significance becomes $\sim 3 \sigma$. For calculating the significance, we apply Excess $=($ signal $-\mathrm{SS}-$ $\mathrm{BRN}-\mathrm{SM}) / \sqrt{2 \mathrm{SS}+\mathrm{BRN}+\mathrm{SM}}[40]$. We also calculate the significance from the likelihood ratio test for the DM fit to the excess and find the significance to be 1.98. The SS and BRN backgrounds emerge from measuring beam-on anticoincident events and the simulation from GEANT4, respectively. We use the same systematic uncertainty $\sim 28 \%$ [41], which incorporates flux, form factor, quenching factor, and the signal acceptance uncertainties.

We first attempt to explain the excess with a DM hypothesis, again assuming that the DM scattering is governed by a different mediator. We fit the selected events, varying the associated (effective) coupling constant $\epsilon$ and mediator mass $M^{\prime}$, which is responsible for the interaction between the DM and the nucleus. The left panel of Fig. 2 shows $1 \sigma$ best fits to the dataset with the cuts implemented (blue band). The scattered "islands" come from our Markov chain Monte Carlo sampling methods, with limited live points that cause some islands in the result. For comparison, the orange band shows the parameter space when performing a fit to the full energy and timing data at $1 \sigma$. We see that there exists an overlapping region and further find that both "before-cut" and "after-cut" datasets are well accommodated by the parameter points with $M^{\prime} \gtrsim 100 \mathrm{MeV}$. For comparison to the DM case, we determine whether a NSI neutrino hypothesis is able to fit both the before-cut and after-cut data. For the neutrino case, we consider a nonzero coupling $g_{e}$, the NSIs in the $\nu_{e}$ neutral-current interaction. As shown in the right panel of Fig. 2, it is not possible to simultaneously fit both the before and after-cut datasets with this neutrino hypothesis. In fact, this NSI model does not show a good fit for the excess in the prompt timing bin (i.e., $T<1.5 \mu \mathrm{s}$ ). The fuzzy region at low $g_{e}$ shows that there is some statistical consistency with the SM in this region, in particular for the before-cut data. The situation becomes even worse with $g_{\mu} \neq 0$, since it affects not only the delayed but also the prompt spectrum.
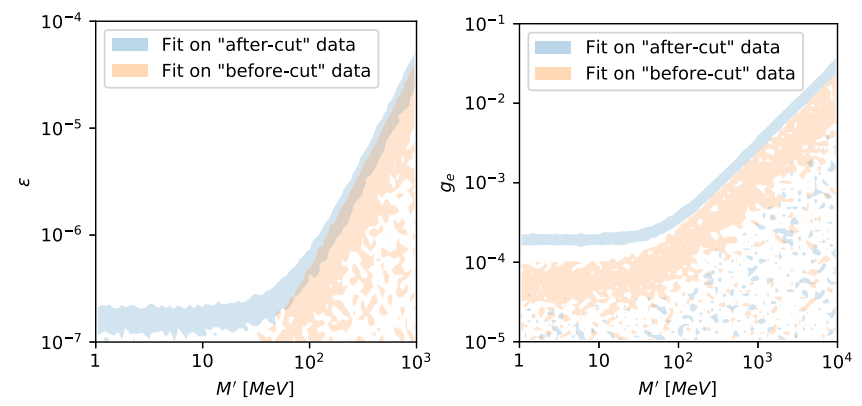

FIG. 2. $1 \sigma$ best fits to the before-cut data (orange) and the aftercut data (blue) for a DM interpretation (left) and a neutrino NSI interpretation (right). 
In the DM case, the parameter $\epsilon$ is defined as $\epsilon=$ $\epsilon_{1}^{q} \epsilon_{2}^{q} \epsilon_{D} \sqrt{\mathrm{BR}_{A^{\prime} \rightarrow x \chi}}$, where $\epsilon_{1}^{q}$ is the $q-A^{\prime}$ kinetic mixing that describes the dark photon production from the $\pi^{-}$absorption, $\epsilon_{2}^{q}$ is the quark-mediator kinetic mixing for the DM-nucleus scattering cross-section, and $g_{D}=e \epsilon_{D}$ is the DM-mediator coupling. This is the most general description, since in a realistic model there can be more than one mediator; e.g., scalar and gauge boson mediators commonly occur in models with spontaneous symmetry breaking. Of course, the best-fit contour can also be interpreted in the case where there exists only a single mediator, i.e., $M^{\prime}=m_{A^{\prime}}$.

The parameter choices that we use to obtain the best-fit points are $\tau_{A^{\prime}}=1 \mathrm{~ns}, m_{A^{\prime}}=75 \mathrm{MeV}$, and $m_{\chi}=5 \mathrm{MeV}$. However, we find that the best-fit points do not change in the $\epsilon-M^{\prime}$ plane for the following variations: (i) for $\tau_{A^{\prime}} \lesssim 4 \mathrm{~ns}$, since the DM flux maximizes for $\tau_{A^{\prime}} \lesssim 4 \mathrm{~ns}$ with $m_{A^{\prime}}<138 \mathrm{MeV}$, (ii) for the nonrelativistic case, i.e., $m_{A^{\prime}}=138 \mathrm{MeV}$, with $\tau_{A^{\prime}} \lesssim 30 \mathrm{~ns}$, and (iii) for any $m_{\chi}$ smaller than $m_{A^{\prime}} / 2$. For relativistic scenarios with large $\tau_{A^{\prime}}$ ( $\geq 4 \mathrm{~ns}$ ), the best-fit regions get scaled by the appropriate associated DM flux (see the top panel of Fig. 1). For nonrelativistic scenarios with large $\tau_{A^{\prime}}(\geq 30 \mathrm{~ns})$, it is not possible to fit before- and after-cut datasets simultaneously because DM will contribute to both before $1 \mu \mathrm{s}$ and after $1 \mu \mathrm{s}$ events. Figure 2 is shown for $R_{n}=4.7 \mathrm{fm}$. However, the best-fit contours do not change for $R_{n}=5.5 \mathrm{fm}$.

Based on the above discussions, we describe the best-fit parameters for the following two scenarios.

(1) Single-mediator scenario: In this case, $\epsilon^{q} \equiv \epsilon_{1}^{q}=\epsilon_{2}^{q}$ and the dark photon $A^{\prime}$ should decay fast. Otherwise, $\epsilon$ is small, meaning that the DM-nucleus scattering is so small that a very small number of events would occur. Here $\epsilon=\epsilon_{1}^{q} \epsilon_{2}^{q} \epsilon_{D} \sqrt{\mathrm{BR}_{A^{\prime} \rightarrow \chi x}} \rightarrow\left(\epsilon^{q}\right)^{2} \epsilon_{D} \sqrt{\mathrm{BR}_{A^{\prime} \rightarrow \chi x}}$. We can choose $\epsilon_{D}=1 / e$ to make $g_{D}=1$, which makes $\tau_{A^{\prime}}$ small, and we can still make use of the left panel of Fig. 2 (where $\tau_{A^{\prime}}$ is set to be $\leq 1 \mathrm{~ns}$ ). Table I shows the best $\epsilon^{q}$ for a few $M^{\prime}\left(=m_{A^{\prime}}\right)$ values, for which the resulting branching ratios for $\pi^{0} \rightarrow \gamma A^{\prime}$ and $\pi^{ \pm} \rightarrow e^{ \pm} \nu A^{\prime}$ agree with current precision data of $\pi^{0}$ and $\pi^{ \pm}$[42]. We do not report any numbers below $M^{\prime}=50 \mathrm{MeV}$ as we find that the best-fit region with the before-cut data does not overlap with that with the after-cut data.

(2) Multimediator scenario: Unlike the previous scenario, $\tau_{A^{\prime}}$ is not necessarily small, since $\chi$ scatters off the target nucleus via a new mediator with large coupling, while the dark photon can decay to a pair of DM particles with a longer lifetime. Table I for a single-mediator scenario still holds with $\epsilon^{q}$ identified as $\sqrt{\epsilon_{1}^{q} \epsilon_{2}^{q} \epsilon_{D} e}$.

$\epsilon^{q}$ 's shown in Table I are obtained assuming that the dark photon couplings to up and down quarks are proportional to their charges. If, however, we want to use the universal charge (e.g., 1), then we need to scale the $\epsilon^{q}$ by $\sqrt{2 Z /(9 A)}$, where $Z=54$ and $A=130$ for CsI. The best-fit values of
TABLE I. Best-fit $\epsilon^{q}$ for a few $M^{\prime}$ values (in $\mathrm{MeV}$ ) for the single-mediator scenario.

\begin{tabular}{ccccc}
\hline \hline$M^{\prime}$ & 50 & 75 & 100 & 1000 \\
\hline$\epsilon^{q}$ & $3.5 \times 10^{-4}$ & $4.4 \times 10^{-4}$ & $5.5 \times 10^{-4}$ & $4.6 \times 10^{-3}$ \\
\hline \hline
\end{tabular}

$\epsilon^{q}$ are below any existing bounds [43,44] arising from meson decays, e.g., $K \rightarrow \pi+$ invisibles [45]. The model details become important for this constraint, i.e., whether it contains fully conserved current, additional Higgs sector [46], or the value of $g_{D}$, etc. The excess can be explained in the allowed regions of parameter space of the realistic models, e.g., $\mathrm{U}(1)_{T 3 R}, \mathrm{U}(1)_{B-L}$, etc., [9,29]. For example, for $50 \mathrm{MeV}$ dark photon, the coupling $(e \epsilon) \sim 10^{-4}$, needed to explain the excess, is well allowed by all the existing data in a generic vector-portal DM model [29]. The COHERENT limit for NSIs of neutrinos is better than any existing limit from various experiments using the timing plus energy data, where the SM backgrounds cannot be sufficiently suppressed [28]. However, for the DM analysis, since we have vetoed the SM neutrino backgrounds using the energy and timing cuts, we can obtain an even better reach in terms of new physics coupling. Now assuming no excess above the backgrounds (which could be caused by an improper estimation of the time of creation of the pions at the target [47]), the values of $\epsilon$ become smaller by a factor of 1.5 compared to those in Table I, as shown in Fig. 3.

The future LDMX experiment [48] will investigate the sub-GeV DM parameter space that arises from a dark photon decaying to DM, using an electron beam dump. We note that this parameter space is already being probed via nuclear recoils at COHERENT, therefore representing a complementary approach. In Fig. 3, we compare the reach of $\left(\epsilon^{X}\right)^{2}$ as a function of mediator mass for the current COHERENT data and for a future argon detector with the LDMX reach assuming that $\epsilon^{q}=\epsilon^{e}$. We also show the

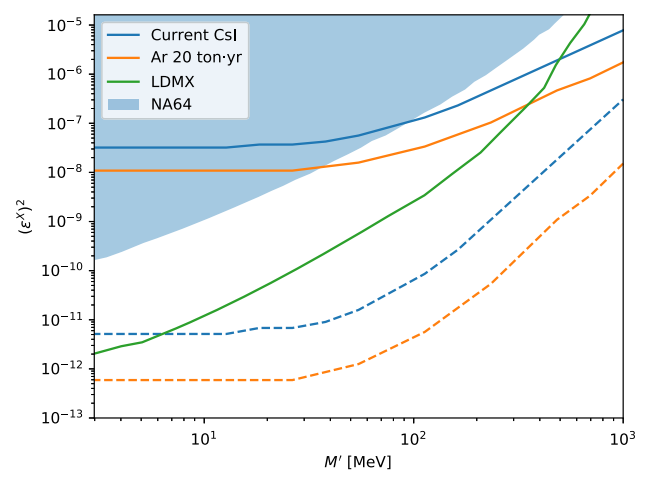

FIG. 3. The coupling $\left(\epsilon^{X}\right)^{2}$ for mediator-nucleus coupling is shown as a function of $M^{\prime}$. The solid (dashed) lines assume $\epsilon^{X}=$ $\epsilon_{1}^{q}=\epsilon_{2}^{q}\left(\epsilon^{X}=\epsilon_{1,2}^{q}\right.$ with $\left.\epsilon_{2,1}^{q}=10^{-2}\right) . \epsilon^{X}$ for LDMX can be understood as $\epsilon$ in Ref. [48]. 
existing limits from NA64 [49] relevant to both single- and multimediator scenarios in the plot assuming $\epsilon^{q}=\epsilon^{e}$.

Our current and projected limits are derived using the formalism of Ref. [22], and they are essentially governed by the $\pi^{0}$ contribution. We show two scenarios: (i) the dark photon coupling $\left(\epsilon_{1}^{q}\right)$ is the same as the mediator-nucleus coupling $\left(\epsilon_{2}^{q}\right)$, and (ii) $\epsilon_{1}^{q}$ is fixed at $10^{-2}$ (currentexperimental constraint [45]) with $\alpha_{D} \equiv g_{D}^{2} /(4 \pi)=0.5$. We use a dark photon mass $m_{A^{\prime}}=75 \mathrm{MeV}$ and a DM mass $m_{\chi}=5 \mathrm{MeV}$. The figure, however, is unchanged for $m_{A^{\prime}} \leq 138 \mathrm{MeV}$, $m_{\chi} \leq m_{A^{\prime}} / 2$, and $\tau_{A^{\prime}} \leq 4 \mathrm{~ns}$. We also note that the reach of the current COHERENT data in probing $\left(\epsilon^{X}\right)^{2}$ in Fig. 3 is competitive with DUNE experiment reach [50].

In conclusion, we have argued that the timing information available in neutrino experiments with pulsed beam such as the COHERENT data is a powerful probe of new physics. We have shown that the combination of energy and timing cuts can eliminate SM neutrino events very efficiently, thereby allowing the possibility of isolating DM-induced events. As applied to the published COHERENT data, we find a considerable number of excess events over the expected backgrounds. This excess of events may be explained by a dark matter hypothesis and is unlikely to be explained by SM neutrino interactions. We note that this conclusion is distinct from the results presented in Ref. [28], where it was showed that, using the full energy and timing data without using the cuts, a neutrino model is able to explain the data. Even though we have presented a DM interpretation of the COHERENT data, it remains possible that the events may be explained by an unidentified background, by a systematic uncertainty on the observed steadystate background, or by exotic beyond the SM scenarios. Distinguishing a background hypothesis from a DM hypothesis may be possible with timing and energy information on individual nuclear recoil events. Our analysis strategy can be used to understand dark photon decaying to DM in similar COHERENT-type setups with timing measurements [51], e.g., J-PARC Sterile Neutrino Search $\left(\mathrm{JSNS}^{2}\right)$ [52] and Coherent Captain-Mills (CCM) [53].

We thank Phil Barbeau, Pilar Coloma, Yuri Efremenko, Pedro Machado, Grayson Rich, and Kate Scholberg for useful discussions. B. D., D. K., and L. E. S. acknowledge support from DOE Award No. de-sc0010813. The work of D. K. was supported in part by the Department of Energy under Award No. DE-FG02-13ER41976/DE-SC0009913. S. L. acknowledges support from College of ScienceStrategic Transformative Research Program (TAMU). The work of J. C.P. is supported by the National Research Foundation of Korea (NRF-2019R1C1C1005073 and NRF-2018R1A4A1025334). The work of S. S. is supported by the National Research Foundation of Korea (NRF2017R1D1A1B03032076). This work was supported by IBS under the project code IBS-R018-D1 (S. S.). *dutta@physics.tamu.edu

†doojin.kim@tamu.edu

\#ikaros@physics.tamu.edu

jcpark@cnu.ac.kr

"sshin@jbnu.ac.kr

"strigari@tamu.edu

[1] E. Aprile et al. (XENON Collaboration), Phys. Rev. Lett. 121, 111302 (2018).

[2] M. Battaglieri et al., in U.S. Cosmic Visions: New Ideas in Dark Matter College Park, MD, USA, 2017 (2017), http://lss.fnal .gov/archive/2017/conf/fermilab-conf-17-282-ae-ppd-t.pdf.

[3] J.-H. Huh, J. E. Kim, J.-C. Park, and S. C. Park, Phys. Rev. D 77, 123503 (2008).

[4] M. Pospelov, A. Ritz, and M. B. Voloshin, Phys. Lett. B 662, 53 (2008).

[5] D. Hooper and K. M. Zurek, Phys. Rev. D 77, 087302 (2008).

[6] C. Cheung, J. T. Ruderman, L.-T. Wang, and I. Yavin, Phys. Rev. D 80, 035008 (2009).

[7] R. Essig, J. Kaplan, P. Schuster, and N. Toro, arXiv: 1004.0691.

[8] R. Essig et al., in Proceedings, 2013 Community Summer Study on the Future of U.S. Particle Physics: Snowmass on the Mississippi (CSS2013): Minneapolis, MN, USA, 2013 (2013), http://www.slac.stanford.edu/econf/C1307292/docs/ IntensityFrontier/NewLight-17.pdf.

[9] B. Dutta, S. Ghosh, and J. Kumar, Phys. Rev. D 100, 075028 (2019).

[10] D. Akimov et al. (COHERENT Collaboration), arXiv: 1803.09183.

[11] T. Ohlsson, Rep. Prog. Phys. 76, 044201 (2013).

[12] O. G. Miranda and H. Nunokawa, New J. Phys. 17, 095002 (2015).

[13] P. Coloma, P. B. Denton, M. C. Gonzalez-Garcia, M. Maltoni, and T. Schwetz, J. High Energy Phys. 04 (2017) 116.

[14] P. Coloma, M. C. Gonzalez-Garcia, M. Maltoni, and T. Schwetz, Phys. Rev. D 96, 115007 (2017).

[15] J. Liao and D. Marfatia, Phys. Lett. B 775, 54 (2017).

[16] J. B. Dent, B. Dutta, S. Liao, J. L. Newstead, L. E. Strigari, and J. W. Walker, Phys. Rev. D 97, 035009 (2018).

[17] J. Billard, J. Johnston, and B. J. Kavanagh, J. Cosmol. Astropart. Phys. 11 (2018) 016.

[18] M. Lindner, W. Rodejohann, and X.-J. Xu, J. High Energy Phys. 03 (2017) 097.

[19] Y. Farzan, M. Lindner, W. Rodejohann, and X.-J. Xu, J. High Energy Phys. 05 (2018) 066.

[20] V. Brdar, W. Rodejohann, and X.-J. Xu, J. High Energy Phys. 12 (2018) 024.

[21] D. Aristizabal Sierra, V. De Romeri, and N. Rojas, Phys. Rev. D 98, 075018 (2018).

[22] A. Datta, B. Dutta, S. Liao, D. Marfatia, and L. E. Strigari, J. High Energy Phys. 01 (2019) 091.

[23] T. S. Kosmas, D. K. Papoulias, M. Tortola, and J. W. F. Valle, Phys. Rev. D 96, 063013 (2017).

[24] C. Blanco, D. Hooper, and P. Machado, arXiv:1901.08094.

[25] E. Ciuffoli, J. Evslin, Q. Fu, and J. Tang, Phys. Rev. D 97, 113003 (2018).

[26] D. Aristizabal Sierra, J. Liao, and D. Marfatia, J. High Energy Phys. 06 (2019) 141. 
[27] D. K. Papoulias, T. S. Kosmas, R. Sahu, V. K. B. Kota, and M. Hota, Phys. Lett. B 800, 135133 (2020).

[28] B. Dutta, S. Liao, S. Sinha, and L. E. Strigari, Phys. Rev. Lett. 123, 061801 (2019).

[29] P. deNiverville, M. Pospelov, and A. Ritz, Phys. Rev. D 92 , 095005 (2015).

[30] S.-F. Ge and I. M. Shoemaker, J. High Energy Phys. 11 (2018) 066.

[31] See Supplemental Material at http://link.aps.org/supplemental/ 10.1103/PhysRevLett.124.121802 for details of the COHERENT experiment and estimations of dark photon angular and energy distributions.

[32] D. Akimov et al. (COHERENT Collaboration), arXiv: 1804.09459.

[33] R. Rapp (COHERENT Collaboration) (private communication).

[34] B. Holdom, Phys. Lett. 166B, 196 (1986).

[35] F. del Aguila, G. D. Coughlan, and M. Quiros, Nucl. Phys. B307, 633 (1988); B312, 751(E) (1989).

[36] K. S. Babu, C. F. Kolda, and J. March-Russell, Phys. Rev. D 57, 6788 (1998).

[37] S. Agostinelli et al. (GEANT4 Collaboration), Nucl. Instrum. Methods Phys. Res., Sect. A 506, 250 (2003).

[38] M. Cadeddu, C. Giunti, Y. F. Li, and Y. Y. Zhang, Phys. Rev. Lett. 120, 072501 (2018).

[39] Unless $m_{A^{\prime}} \approx 138 \mathrm{MeV}$ and $\tau_{A^{\prime}} \gtrsim 0.03 \mu \mathrm{s}$.

[40] K. Scholberg (COHERENT Collaboration), Proc. Sci. NuFact2017 (2018) 020.
[41] D. Akimov et al. (COHERENT Collaboration), Science 357, 1123 (2017).

[42] M. Tanabashi et al. (Particle Data Group), Phys. Rev. D 98, 030001 (2018).

[43] R. Essig, J. Mardon, M. Papucci, T. Volansky, and Y.-M. Zhong, J. High Energy Phys. 11 (2013) 167.

[44] J. A. Dror, R. Lasenby, and M. Pospelov, Phys. Rev. D 96, 075036 (2017).

[45] A. V. Artamonov et al. (BNL-E949 Collaboration), Phys. Rev. D 79, 092004 (2009).

[46] H. Davoudiasl, H.-S. Lee, and W. J. Marciano, Phys. Rev. D 89, 095006 (2014).

[47] Kate Scholberg, the COHERENT Collaboration is investigating this possibility (private communication).

[48] T. Åkesson et al. (LDMX Collaboration), arXiv:1808 .05219 .

[49] D. Banerjee et al., Phys. Rev. Lett. 123, 121801 (2019).

[50] V. De Romeri, K. J. Kelly, and P. A. N. Machado, Phys. Rev. D 100, 095010 (2019).

[51] As far as the beam pulse duration is less than $\sim 1 \mu \mathrm{s}$ to populate DM signals in the prompt timing bins and the pulse interval is greater than $\sim 10 \mu$ s to sufficiently attenuate the flux of delayed neutrinos.

[52] S. Ajimura et al., arXiv:1705.08629.

[53] A. A. Aguilar-Arevalo et al. (2018), https://p25ext.lanl.gov/ lee/CaptainMills/Documentation/CCM_Proposal_Lujan_ 2018_V1.pdf. 